\title{
Turing patterns in a single-step autocatalytic reaction
}

\author{
Dezsö Horváth and Ágota Tóth \\ Department of Physical Chemistry, József Attila University, P.O. Box 105, Szeged, H-6701, \\ Hungary
}

Stable Turing patterns are presented in the simplest reaction-diffusion system containing a single autocatalytic step in a continuously fed unstirred reactor. In the one-variable homogeneous system exhibiting only bistability, the spatial instability arises from the decoupling of the species by unequal diffusion. The results suggest the possibility of experimentally finding Turing instability among the relatively common bistable chemical systems.

In 1952 Turing introduced a novel idea that diffusion may destabilize a state of homogeneously distributed chemical reactants giving rise to spontaneous patterns with large amplitude spatial oscillations in the concentrations of some species. ${ }^{1}$ Several theoretical investigations, following Turing's original work, have shown that in a simple two-variable model the system must exhibit bistability or oscillations and the diffusion coefficients of the components must differ as necessary conditions for spontaneous pattern formation. ${ }^{2}$ The first experimental presentations of Turing structures ${ }^{3}$ have furthermore required the application of a continuously fed unstirred reactor (CFUR), ${ }^{4}$ which has allowed the study of spatially distributed open chemical systems far from equilibrium.

The simplest models showing Turing instability have consisted of a cubic-autocatalytic step and a first-order decay of the autocatalyst (the Gray-Scott model $^{5}$ ) in a CFUR. ${ }^{6}$ This two-step mechanism may exhibit rich dynamic behavior with homogeneous oscillations, ${ }^{5}$ stable and oscillatory patterns, ${ }^{7}$ and even spatiotemporal chaos. ${ }^{8}$

Although the existence of temporal oscillations is not a prerequisite for Turing instability, studies of spatial pattern formation in chemical systems have generally tied Turing patterns to the existence of homogeneous oscillations ${ }^{9-11}$ and overlooked simple autocatalytic reactions showing only bistability as possible candidates for Turing patterns.

In this paper we show that a single autocatalytic step in a CFUR may exhibit Turing instability and sustain stable patterns. The reactant and the autocatalyst are only spatially decoupled by unequal diffusion, therefore no temporal oscillation of the homogeneous system is possible.

The simplest single autocatalytic step showing bistability in an open system is the cubic autocatalysis

$$
\mathrm{A}+2 \mathrm{~B} \stackrel{k}{\longrightarrow} 3 \mathrm{~B}
$$

for which the reaction-diffusion system in a CFUR is governed by

$$
\begin{aligned}
& \frac{\partial a}{\partial t}=D_{\mathrm{A}} \nabla^{2} a-k a b^{2}+k_{0}\left(a_{0}-a\right) \\
& \frac{\partial b}{\partial t}=D_{\mathrm{B}} \nabla^{2} b+k a b^{2}+k_{0}\left(b_{0}-b\right)
\end{aligned}
$$

where $a$ and $b$ are the concentration of species A and B in the reactor and $a_{0}$ and $b_{0}$ are those in the reservoir with $k_{0}$ representing the coupling between the reactor and the reservoir. By introducing dimensionless variables $\alpha=a / a_{0}$ and $\beta=$ $b / a_{0}$, we can transform eqn. (2) into

$$
\begin{aligned}
& \frac{\partial \alpha}{\partial \tau}=\delta \nabla^{2} \alpha-\alpha \beta^{2}+\kappa(1-\alpha) \\
& \frac{\partial \beta}{\partial \tau}=\nabla^{2} \beta+\alpha \beta^{2}+\kappa\left(\beta_{0}-\beta\right)
\end{aligned}
$$

where $\beta_{0}=b_{0} / a_{0}, \delta=D_{\mathrm{A}} / D_{\mathrm{B}}$ is the ratio of the diffusion coefficients, and $\kappa=k_{0} / k a_{0}^{2}$ is the exchange coefficient. The dimensionless space and time coordinates are scaled as $\xi=$ $x\left(k a_{0}^{2} / D_{\mathrm{B}}\right)^{1 / 2}$ and $\tau=k a_{0}^{2} t$.

The stability analysis of the homogeneous system shows that bistability exists for $\beta_{0}<0.125$ in the region defined as

$$
\frac{\beta_{-}^{3}-\beta_{-}^{2}\left(1+\beta_{0}\right)}{\beta_{0}-\beta_{-}}<\kappa<\frac{\beta_{+}^{3}-\beta_{+}^{2}\left(1+\beta_{0}\right)}{\beta_{0}-\beta_{+}}
$$

with

$$
\beta_{ \pm}=\frac{4 \beta_{0}+1 \pm\left[\left(4 \beta_{0}+1\right)^{2}-16 \beta_{0}\left(\beta_{0}+1\right)\right]^{1 / 2}}{4}
$$

Outside this regime the system has only one stable steadystate solution. In the case of Turing instability the stable steady states of the homogeneous system lose stability to spatial perturbations requiring a positive real part for one of the eigenvalues of the Jacobian matrix $(J)$ constructed from eqn. (3)

$$
J=\left(\begin{array}{cc}
-\beta_{\mathrm{s}}^{2}-\kappa-\delta k^{2} & -2 \alpha_{\mathrm{s}} \beta_{\mathrm{s}} \\
\beta_{\mathrm{s}}^{2} & 2 \alpha_{\mathrm{s}} \beta_{\mathrm{s}}-\kappa-k^{2}
\end{array}\right)
$$

where $\alpha_{\mathrm{s}}$ and $\beta_{\mathrm{s}}$ are the appropriate steady-state solutions of the homogeneous system and $k$ is the wavenumber associated with the spatial perturbation. Since $\operatorname{Tr}(J)<0$ for all stable steady-state solutions of the homogeneous system, Turing instability can only occur if $|J|$ becomes negative for some $k$ yielding

$$
\left(2 \alpha_{\mathrm{s}} \beta_{\mathrm{s}}-\kappa\right) \delta-\beta_{\mathrm{s}}^{2}-\kappa>2\left[\kappa\left(\beta_{\mathrm{s}}^{2}+\kappa-2 \alpha_{\mathrm{s}} \beta_{\mathrm{s}}\right) \delta\right]^{1 / 2}
$$

as a necessary requirement, which further implies that reactant A should diffuse faster than the autocatalyst, i.e., $\delta>$ $\delta_{\text {cr }}>1$. As shown in Fig. 1 for $\delta=10$, Turing bifurcation appears at the saddle-node bifurcation at the end of the flow branch at $\kappa=0.32338$ then slightly widens the range of instability (see inset in Fig. 1) with increasing $\kappa$. After turning back in the monostable regime, it nearly parallels the saddle-node bifurcation at the end of the thermal branch. In the bistable region the Turing space is wider on the thermal branch indicating that steady states preceding the saddle-node bifurcation on this branch lose stability to spatial perturbation at lower $\delta_{\mathrm{cr}}$; and in fact, the minimum $\delta_{\mathrm{cr}}=2$ is found as $\beta_{0} \rightarrow 0$ and $\kappa \rightarrow 0.25$.

The existence of Turing instability overall extends the bistable regime with the introduction of stable patterns. 


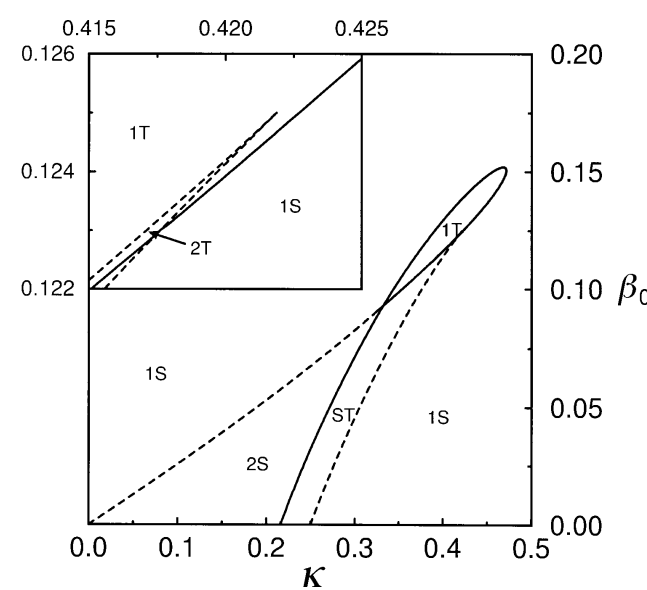

Fig. 1 Bifurcation diagram for $\delta=10$ with dashed lines representing saddle-node bifurcations and the solid lines Turing bifurcations. The different regions are indicated as: one homogeneous stable steady state (1S); two homogeneous stable steady states (2S); one homogeneous stable steady state unstable to spatial perturbations (1T); two homogeneous stable steady states with one unstable to spatial perturbations (ST); two homogeneous stable steady states unstable to spatial perturbations (2T).

Without loss of generality this may readily be demonstrated with the construction of a hysteresis diagram in a finite onedimensional system with $\nabla^{2}=\partial^{2} / \partial \xi^{2}$ and imposing no-flux boundary conditions at the ends

$$
\frac{\partial \alpha}{\partial \xi}=\frac{\partial \beta}{\partial \xi}=0 \quad \text { at } \quad \xi=0 \quad \text { and } \quad \xi=L
$$

At constant $\beta_{0}=0.121, \kappa$ is increased from the homogeneous solution on the thermal branch until the stable homogeneous solution on the flow branch is reached then decreased back to obtain the initial homogeneous state as shown in Fig. 2. During the calculations a stable pattern is perturbed with normally distributed random noise and a numerical integration using the CVODE package ${ }^{12}$ is carried out to obtain the new stable solution. Parameter $\kappa$ is then incremented or decremented with $4 \times 10^{-4}$ and the procedure is repeated.

On increasing $\kappa$ from 0.3824 to 0.3828 the homogeneous steady state loses stability through a supercritical Turing bifurcation giving rise to a stable pattern. Among all spatial modes satisfying the boundary conditions only the one with $n=9$ has a positive eigenvalue, where $n$ represents the number of half-wavelengths. This mode then grows out of the random noise around the homogeneous steady state with the final pattern retaining the wavelength and amplitude of the active mode in the vicinity of the bifurcation

$$
\left(\begin{array}{c}
\alpha \\
\beta
\end{array}\right)=\left(\begin{array}{c}
\alpha_{\mathrm{s}} \\
\beta_{\mathrm{s}}
\end{array}\right) \pm\left(\frac{\kappa-\kappa_{\mathrm{c}}}{\phi}\right)^{1 / 2}\left(\begin{array}{l}
1 \\
C
\end{array}\right) \cos \left(\frac{n_{\mathrm{c}} \pi}{L} \xi\right)
$$

where

$$
\kappa_{\mathrm{c}}=0.3824691835 \text {, }
$$
$C=-2.555955806$, and $n_{\mathrm{c}}=9$. At $\kappa=0.4356$ a shift in the wavelength occurs and a pattern with $n=8$ evolves which remains stable up to $\kappa=0.4424$, where a new mode with $n=4$ is formed. Finally, at $\kappa=0.4480$ the homogeneous steady state on the flow branch is attained.

On decreasing $\kappa$ from 0.4128 to 0.4124 the homogeneous steady state loses stability through a subcritical Turing bifurcation with active mode

$$
\left(\begin{array}{c}
\alpha \\
\beta
\end{array}\right)=\left(\begin{array}{c}
\alpha_{\mathrm{s}} \\
\beta_{\mathrm{s}}
\end{array}\right) \pm\left(\frac{\kappa_{\mathrm{c}}-\kappa}{\phi}\right)^{1 / 2}\left(\begin{array}{l}
1 \\
C
\end{array}\right) \cos \left(\frac{n_{\mathrm{c}} \pi}{L} \xi\right)
$$

where $\quad \kappa_{\mathrm{c}}=0.4125164327$, $\phi=-1.84405933$, $C=-1.331822821$, and $n_{\mathrm{c}}=4$. This mode develops initially from the random noise imposed on the homogeneous state;
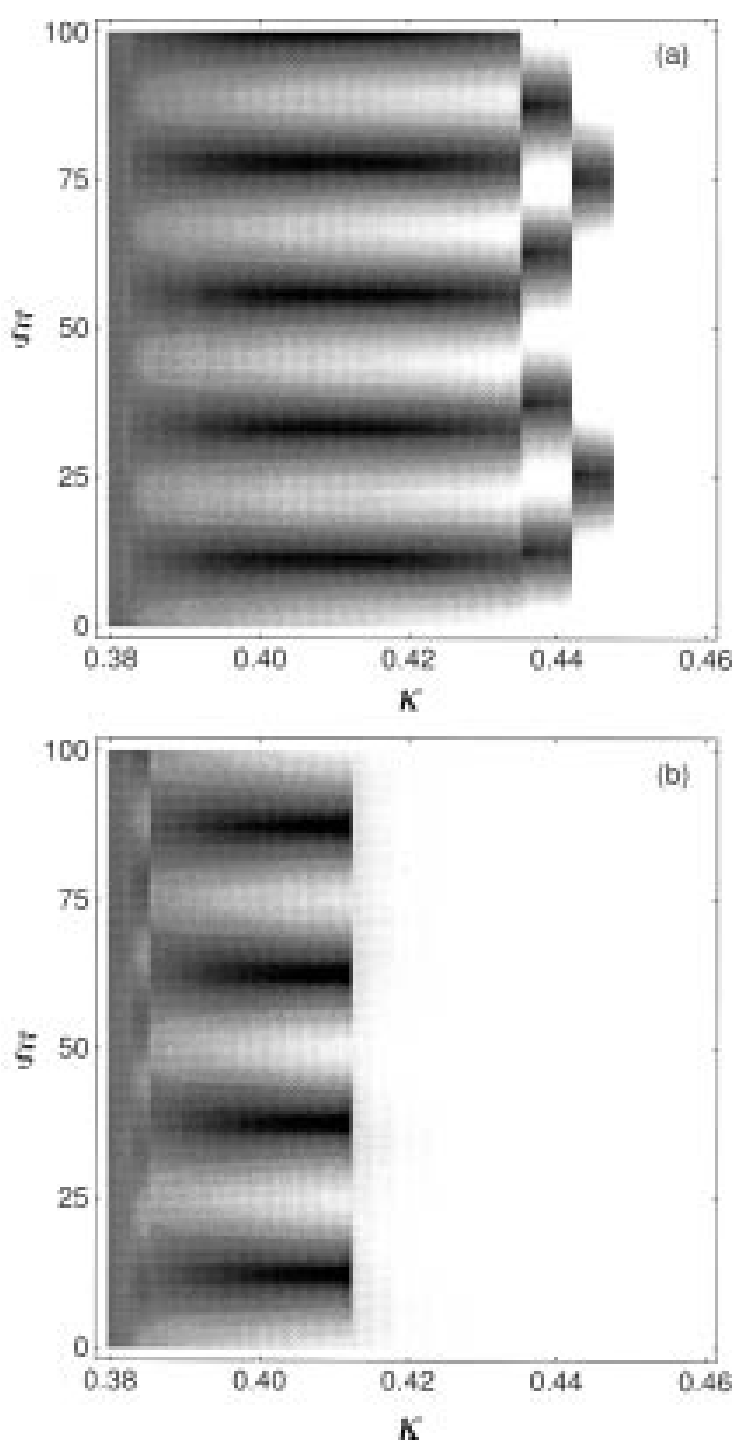

Fig. 2 Hysteresis diagram showing the calculated stable patterns as the exchange coefficient $\kappa$ is increased (a) and decreased (b). The dimensionless concentration $\beta$ is represented by a gray scale with black corresponding to the maximum value of $\beta$ and white to the minimum. The parameters used in the calculations are $\beta_{0}=0.121$, $\delta=10$. The numerical integration is carried out on a 1001-point grid with spacing $h=0.1$.

however, as it leaves the neighborhood of the steady state it evolves into the mode with $n=8$ corresponding to that observed for $0.4356 \leqslant \kappa \leqslant 0.4420$ with increasing $\kappa$. This pattern is stable with monotonously decreasing amplitude down to $\kappa=0.3852$, where a wavelength shift results in the formation of the mode with $n=9$. At this point the hysteresis loop is closed, as by further decreasing $\kappa$ the system exhibits the same behavior as previously with increasing $\kappa$. The final homogeneous steady state regains stability at $\kappa=0.3824$.

The regions of stability for the various patterns in the hysteresis cycle are summarized in Fig. 3. The new spatial mode with $n=6$ can be obtained from the $n=4$ mode by decreasing $\kappa$. When this pattern loses stability, it evolves into the mode with $n=8$ by decreasing $\kappa$ or yields the homogeneous state on the flow branch by increasing $\kappa$.

In experimental studies the effective diffusion coefficient of the autocatalyst has generally been lowered by binding it to an immobile species. ${ }^{3,10}$ Lengyel and Epstein have shown that this complex formation shifts the Hopf bifurcation point beyond the Turing bifurcation point so that Turing instability arises between them. ${ }^{11}$ This general method, however, fails in our system, where instability to spatial perturbations occurs 


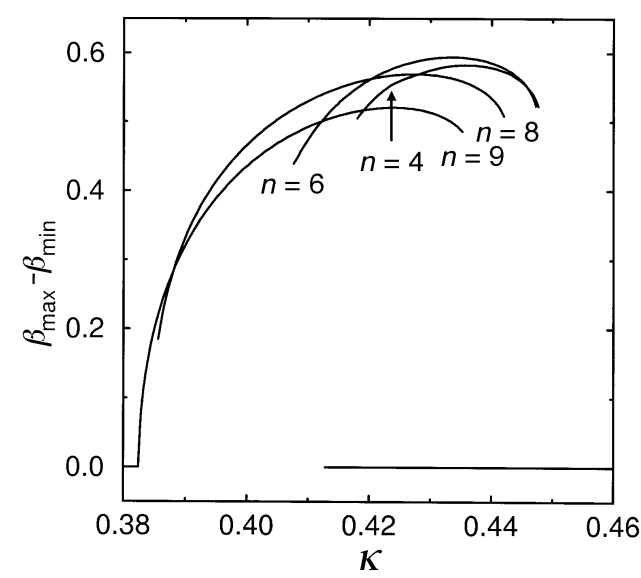

Fig. 3 Amplitude of the stable spatial patterns at $\beta_{0}=0.121$. The parameters are the same as in Fig. 2.

near a saddle-node bifurcation. The saddle-node bifurcation is unaffected by the complex formation as the latter does not change the sign of the determinant of the Jacobian for homogeneous unstable saddle points; thus, the inequality of the actual diffusion coefficients remains a necessary requirement in our system for observing spontaneous Turing patterns.

In this work we have shown that a single autocatalytic step in a CFUR may give rise to Turing patterns. We have numerically investigated the stability of the obtained patterns in the simplest, cubic-autocatalytic system. The spatial decoupling of the two components by unequal diffusion leads to a bifurcation diagram where the location of the spatial oscillations of Turing structures are similar to that of temporal oscillations in the cross-shaped diagrams of chemical systems in a continuously stirred tank reactor. ${ }^{13} \mathrm{~A}$ recent study on the FitzHugh-Nagumo model has also shown a similar crossshaped diagram in the absence of Hopf bifurcation. ${ }^{14}$ Based on these bifurcation diagrams one may experimentally search for stable Turing structure in the pool of simple autocatalytic reactions exhibiting only bistability.

We thank Dr Vilmos Gáspár for critically reading the manuscript. This work was supported by the Hungarian Science
Foundation (OTKA F022037). D.H. is grateful to the Foundation for Hungarian Higher Education and Research for Magyary Zoltán Fellowship. A.T. thanks the Hungarian Science Foundation (OTKA D24071) for support.

\section{References}

1 A. M. Turing, Philos. Trans. R. Soc. London B, 1952, 237, 37

2 G. Nicolis and I. Prigogine, Self-Organization in Nonequilibrium Chemical Systems, Wiley, New York, 1977; H. Haken, Synergetics, An Introduction, Springer-Verlag, Berlin, 1977; J. D. Murray, Mathematical Biology, Springer-Verlag, Berlin, 1989; V. Dufiet and J. Boissonade, J. Chem. Phys., 1992, 98, 664.

3 V. Castets, E. Dulos, J. Boissonade and P. De Kepper, Phys. Rev. Lett., 1990, 64, 2953; Q. Ouyang and H. L. Swinney, Nature (London), 1991, 352, 610.

4 Z. Noszticzius, W. Horsthemke, W. D. McCormick, H. L. Swinney and W. Y. Tam, Nature (London), 1987, 329, 619.

5 P. Gray and S. K. Scott, Chem. Eng. Sci., 1984, 39, 1087.

6 J. A. Vastano, J. E. Pearson, W. Horsthemke and H. L. Swinney, Phys. Lett. A, 1987, 124, 320; J. A. Vastano, J. E. Pearson, W. Horsthemke and H. L. Swinney, J. Chem. Phys., 1988, 88, 6175.

7 W. N. Reynolds, J. E. Pearson and S. Ponce-Dawson, Phys. Rev. Lett., 1994, 72, 2797; K. Lee, W. D. McCormick, J. E. Pearson and H. L. Swinney, Nature (London), 1994, 369, 215.

8 J. H. Merkin, V. Petrov, S. K. Scott and K. Showalter, J. Chem. Soc., Faraday Trans., 1996, 92, 2911.

9 J. Boissonade, E. Dulos and P. DeKepper, in Chemical Waves and Patterns, ed. R. Kapral and K. Showalter, Kluwer, Dordrecht, 1995, pp. 221-268.

10 I. Lengyel and I. R. Epstein, Acc. Chem. Res., 1993, 26, 235.

11 I. Lengyel and I. R. Epstein, Proc. Natl. Acad. Sci. USA, 1992, 89, 3977.

12 P. N. Brown, G. D. Byrne and A. C. Hindmarsh, SIAM J. Sci. Stat. Comput., 1989, 10, 1038

13 P. De Kepper and J. Boissonade, in Oscillations and Traveling Waves in Chemical Systems, ed. R. J. Field and M. Burger, WileyInterscience, New York, 1985, pp. 223-256.

14 S. Métens, G. Dewel, P. Borckmans and R. Engelhardt, Europhys. Lett., 1997, 37, 109.

Paper 7/05895K; Received 12th August, 1997 